\title{
Missing Data and Multiple Imputation Decision Tree
}

\author{
Created by \\ Adrienne D. Woods, Pam Davis-Kean, Max Halvorson, Kevin King, Jessica R. Logan, \\ Menglin Xu, Sierra Bainter, Denver Brown, James M. Clay, Rick A. Cruz, Mahmoud M. \\ Elsherif, Daria Gerasimova, Keven Joyal-Desmarais, David Moreau, Jayson Nissen, \\ Kathleen Schmidt, Alex Uzdavines, Ben Van Dusen, Martin Vasilev \\ \& \\ all those who participated in the SIPS 2021 virtual hackathon \\ "Best Practices for Addressing Missing Data through Multiple Imputation" \\ https://osf.io/j3f8m/
}

\section{Suggested Citation:}

Woods, A. D., Davis-Kean, P., Halvorson, M., King, K. M., Logan, J. R., Xu, M., ... \& Vasilev, M. (2021). Missing Data and Multiple Imputation Decision Tree.

PsyArXiv. https://doi.org/10.31234/osf.io/mdw5r

Is something missing or not quite right? Tell us!

We'll do our best to update this document with new information or additional FAQs on a rolling basis. Please fill out this Google form: https://forms.gle/GreNaNZ94tMTkCb68 


\section{Authors Note:}

This document is intended to provide practical guidelines for researchers to follow when examining their data for missingness and making decisions about how to handle that missingness. We primarily offer recommendations for multiple imputation, but also indicate where the same decisional guidelines are appropriate for other types of missing data procedures such as full information maximum likelihood (FIML). Note that this is an ideal flow. In practice, the decision process for missing data analysis and multiple imputation is very non-linear. You might realize there was something you didn't think about in Step 1 when checking your trace plots (Step 5) that would impact the entire process. This is completely normal; documenting what you did and why you did it (even a need to go back to an earlier step!) is helpful for people going back and looking at your work later. We are indebted to the following individuals for their similar work, which helped us form the basis for this decision tree:

Logan, J. R. [@jarlogan]. (2021, March 3). First, the distinction between missing not at random, missing at random, and missing completely at random are theoretical... [Tweet Thread]. Twitter. https://twitter.com/jarlogan/status/1367162664972075009

Manly, C. A. \& Wells, R. S. (2015). Reporting the use of multiple imputation for missing data in higher education research. Research in Higher Education, 56, 397-409. https://doi.org/10.1007/s11162-014-9344-9 


\section{Table of Contents}

Step 1: Theorize about your Potential Missingness $\quad 6$

$\begin{array}{ll}\text { When should you theorize about your potential missingness? } & 6\end{array}$

$\begin{array}{ll}\text { Think about why data may be missing in your sample } & 6\end{array}$

$\begin{array}{ll}\text { Why does the missingness pattern matter? } & 7\end{array}$

$\begin{array}{ll}\text { What are the three different missingness patterns? } & 7\end{array}$

1. MCAR (Missing Completely at Random)

2. MAR (Missing at Random) 8

3. MNAR (Missing Not at Random) 9

Suggested next steps based on missingness pattern $\quad 11$

If you think your data could be MCAR, your next steps are...

If you think your data could be MAR, your next steps are...

If you think your data could be MNAR, your next steps are...

Step 2: Pick your Poison - Decide which Method to Use 13

Option A: Deletion Methods 13

1. Listwise Deletion 13

2. Pairwise Deletion 14

Option B: Multiple Imputation $\quad 15$

When your data are MCAR or MAR...

When your data are MNAR... 16

Option C: Full Information Maximum Likelihood (FIML) 16

When your data are MCAR or MAR...

When your data are MNAR...

I've picked my poison. What are my next steps? 17

Step 2.5: Preregister your missing data decisions (optional but strongly recommended)18 When do you preregister your missing data decisions? 18

$\begin{array}{ll}\text { What is preregistration? } & 18\end{array}$

Table 1. Guides and Templates for Pre-Registration 19

$\begin{array}{ll}\text { What should you preregister? } & 20\end{array}$

Why does this matter? $\quad 20$

I've preregistered (yay!). What are my next steps? 20

Step 3: Test for the Missingness Mechanism 21

When should you start testing your data to confirm whether your missingness occurs (not) at random?

Why evaluate patterns in missingness?

1. Examine patterns of missingness - for entire scales, items within scales, patterns of missing data points, groups of people

2. Evaluate how much missingness you have on each variable of interest 
3. Choose a decision rule for determining whether missingness is related to other variables

in your dataset

4. Check whether any auxiliary variables are related to your missingness 22

Test auxiliary variables for inclusion $\quad 23$

I have evaluated my data. Now what do I do?

1. Create a table of which variables are or are not related to missingness on your key variables

2. Decide which auxiliary variables to include in missing data adjustment procedures and retain those variables

\section{Step 4: Data Carpentry - Setting Up Your Data}

When do you set up your data for multiple imputation?

Considerations

Check your distributions

Transform variables prior to imputation

Impute into both your dependent variable(s) and your predictor(s) of interest

Impute categorical data (or, not)

Do not impute data that should be missing

Include weights when appropriate

Nested data considerations

The importance of congeniality

How long will the imputation take?

My data are set up and ready to go! What are my next steps?

Step 5: Multiply Impute Your Data

Choose the type of multiple imputation model 31

Multivariate Normal Imputation (MVN)

Markov chain Monte Carlo (MCMC) 31

Chained (MICE/ICE) $\quad 32$

Set up your MI model 33

Make it reproducible $\quad 33$

Match to your planned analyses 33

Choose the number of imputations $(\mathrm{m}) \quad 33$

Make sure your imputation model worked! 36

Double-check precision $\quad 36$

Check for proper convergence $\quad 36$

$\begin{array}{ll}\text { Look at descriptive information } & 37\end{array}$

Check the goodness-of-fit of the imputation models 38

Assess the predictive ability of your model 38

I have imputed my data and checked that everything went smoothly. Now what do I do? 39

Step 6: Report your Results $\quad 40$

$\begin{array}{ll}\text { Amount (rates) of missing data } & 40\end{array}$ 
Report information about missingness mechanism and why you think this is the case

Report the multiple imputation model setup

Report results from your checks to make sure the imputation worked

Example methods paragraphs

Frequently Asked Questions

What about Little's MCAR test, can't I use that to determine if my data are MCAR? $\quad 44$

Am I making up data or manufacturing results?

I did my analysis once, and then I imputed my data and my significant result went away!

Doesn't that mean I shouldn't use imputation?

But can I impute every variable? Or, are there any variables we should not impute?

Are deletion methods and multiple imputation or FIML the only options?

Table 2. Summary and Errors of Common Missing Data Techniques. 49

Where do I upload my preregistration?

How much missingness is too much?

What software should I use?

Should I use multiple imputation or FIML?

Table 3. Pros and Cons of FIML vs. Multiple Imputation 


\section{Step 1: Theorize about your Potential}

\section{Missingness}

- When should you theorize about your potential missingness?

- You should think about what patterns of missing data may exist in your sample prior to examining the data directly. Use what you know about the data collection procedure or which variables were collected to come up with theories about what type of missingness patterns you might expect to see.

\section{- Think about why data may be missing in your sample}

- Missing data corresponds to three levels of analysis: item-level missingness, construct-level missingness, and person-level missingness.

- Item-level missingness occurs when respondent leaves a few items blank on a multi-item scale. Items can be skipped for several reasons (e.g. items deal with sensitive information such as drug use that participants are reluctant to share).

- Construct-level missingness occurs when respondent answers zero items from a scale (i.e. omitting an entire construct).

- Person-level missingness involves failure by an individual to respond to any part of the survey.

- Here, we focus primarily on person-level missingness, though the imputation approach is similar for item- and construct-level missingness.

- When researchers discuss missing data, they usually make a distinction between three main ways in which data comes to be "missing". These are known as missing completely at random (MCAR), missing at random (MAR), and missing not at random (MNAR) and are each elaborated on below. The distinction between these is largely theoretical, and you can't fully prove one is the case over the other. However, each has important 
implications for what type of missing data tools you should (or shouldn't) use. As a researcher, it is important to think about which of these is most likely in your data, and justify why you think this may be the case. If you struggle with the distinction within your dataset, you can still look ahead in this guide. Sometimes, you can still justify a decision even if you cannot entirely choose between two of these.

\section{- Why does the missingness pattern matter?}

- MNAR, MAR, and MCAR each have different assumptions about how generalizable your data are, and how valid your inferences are.

- If you use listwise deletion, you can only generalize to those people with complete data.

- e.g., so your results only pertain to people who are willing to complete a survey (for example).

- Unfortunately, many researchers consider missing data as part of their analytic plan when it actually needs to be considered as part of the research design. When collecting data you should consider what occurs when you recruit a participant and they do not give you complete data. In order to adequately adjust for missingness we need to have information on additional variables that may be related to the refusal to continue the study or attrition. This may include always asking a set of demographic questions (income, education level, occupation) that are often associated with attrition from studies that can be asked during initial recruitment.

\section{- What are the three different missingness patterns?}

1. MCAR (Missing Completely at Random)

- MCAR refers to a situation when random processes lead to equal probabilities of any given data point to be missing (i.e., the likelihood of missingness is the same for all data points). For example:

- If you created a computer code to select a random subset of your data and convert that data to be missing, this would be MCAR 
- Researchers will sometimes make use of "planned missingness" designs in which each person answering a survey is only given a random subset of the questions to answer. This type of procedure creates missingness (by design) that is MCAR.

- MCAR will also occur if a truly random technical issue arises (e.g., some apparatus in a mouse experiment failed at random times). However, it can be very difficult (and often impossible) to know. Often, issues that at first appear random can actually be systematic (e.g., you later find out that one research assistant in the team made more mistakes calibrating the apparatus than other research assistants).

- Overall, MCAR is commonly seen as "ignorable missingness"--that is, your results will still be unbiased if you simply ignore missing responses and analyse those responses you actually have.

\section{- 2. MAR (Missing at Random)}

- MAR refers to a situation when the probability of data points being missing can be fully accounted for using information from data you have actually observed. Often, MAR can be said to occur if different identifiable groups in your data differ from each other in missingness, but missingness is MAR within the groups. For example:

- If the probability of completing a survey about depression severity is related to sex (e.g., men are less likely to complete the questionnaire about depression than women) but not related to the severity of depression (e.g., more depressed people are less likely to complete the survey), then the data may be regarded as MAR (Mack et al.. 2018) if aside from this difference across sex, the remainder of missing data are random. (If people experiencing more severe depression were less likely to complete the questionnaire about depression, and thus the probability of 
missingness could not be measured with an observed variable in your dataset, the data would be MNAR).

- When making an argument for MAR, you can also consider other known patterns in what predicts who will have missing data. For instance, women might be more likely to have missing data in items from a diet scale, while men might be more likely to have more missing items in a depression scale (Tsiampalis \& Panagiotakos, 2020).

- Like MCAR, MAR can also be considered "ignorable missingness", but only IF--and ONLY IF--your missing data models include the variables that fully account for missingness in your data (i.e., auxiliary variables).

- Most modern methods to account for missing data make an assumption that your missing data is at least MAR. However, adequately accounting for missingness using observed variables can be tricky, so you will need to make a good case for why you think the data are MAR.

- Sometimes, you may need to create or include new variables to satisfy the MAR assumption. For example, based on previous research, you may know that students tend to skip certain questions based on how difficult those items are (e.g., they skip harder items). If you are able to create a variable that measures "level of difficulty" for your questions, and you include it in your missing data model, then you can reasonably assume MAR.

\section{- 3. MNAR (Missing Not at Random)}

- MNAR occurs if the probability of being missing varies in a way that we cannot measure, but only theorize about. Put another way, MNAR can occur if missingness depends not only on the observed data but also on the missing values (Fielding et al., 2008). This is known as "non-ignorable missingness."

- For instance, an individual who has reduced quality of life as a result of treatment side-effects may be less likely to return 
a questionnaire measuring treatment side-effects. In this example, the missing value for treatment side-effects depends on the missing quality of life score. Your analysis of this sample will thus underestimate the prevalence or impact of quality of life score on treatment side-effects.

- However, even though we assume that data are MNAR under this example, understanding and discussing this missingness mechanism can provide informative insight about your research question and processes (for example: foster better attempts to obtain data for patients experiencing lower quality of life) (Fielding et al., 2008).

- There is some disagreement in the field over whether you should adjust for missing data when your data are MNAR.

- On the one hand, with MNAR data, there's nothing you can really do to fully resolve the bias in your sample, and your results must be interpreted in light of this limitation. This is because any estimation or model you run based on MNAR data will be biased toward the individuals who are present in your data. Therefore, even if you use imputation methods, your results will still be biased. For this reason, some researchers choose not to impute when their data are MNAR.

- On the other hand, adjusting for missing data may be a better solution than just throwing out cases under listwise deletion when your data are MNAR. Multiple imputation can still produce less biased estimates than listwise deletion when your data are MNAR, especially when you include a lot of auxiliary variables (van Ginkel et al., 2020).

- There are also additional considerations here that may be applicable to you. When deciding whether to impute MNAR data, it may be relevant to consider the design and the cost of running additional participants. For example, if you have an intervention design that is 
costly to run, or a neuroimaging study, then you might want to maximize the data at hand, even if biased (as long as you are getting more signal than noise doing so), so you choose to impute. If, on the other hand, you are dealing with data that is very easy/cheap to collect (e.g., a quick questionnaire on MTurk), then the cost of imputing 'imperfect' data might be greater than collecting new complete cases with the MNAR mechanism accounted for as a new variable (and so making your data MAR).

- Including auxiliary variables in $\mathrm{Ml}$ can reduce the impact of MNAR missingness and partially restore lost power due to missingness, which would otherwise not be recovered with listwise deletion methods ( et al., 2001; Graham, 2009).

- It is our recommendation that you always adjust for data that are MAR, even if you also have data that are also MNAR. Because you are likely planning to analyze your data anyway, adjusting for MAR missing increases your available sample size and power as well as reduces the non-MNAR bias that is present in your sample.

- Having MNAR data does NOT mean you cannot analyze your data. But you should clearly indicate that your data are likely to be MNAR in your limitations and explain that you cannot generalize beyond your sample given these missingness patterns. Even if you do adjust for other missingness mechanisms, you should always disclose if you think your data are MNAR.

\section{- Suggested next steps based on missingness pattern}

\section{- If you think your data could be MCAR, your next steps are...}

- Step 1a: Pick your poison - choose how you will handle your missing data 


\section{AND/OR}

- Step 1b: Preregister your missing data decisions (optional but strongly recommended)

\section{AND}

- Step 2: Test for missingness mechanism (just to be sure)

- If you think your data could be MAR, your next steps are...

- Step 1: Think about what variables there are in your dataset that could be related to the missingness

AND

- Step 2a: Pick your poison - choose how you will handle your missing data

\section{AND/OR}

- Step 2b: Preregister your missing data decisions (optional but strongly recommended)

\section{AND}

- Step 3: Test for missingness mechanism

- If you think your data could be MNAR, your next steps are...

- Step 1: Write in your missing data method section AND discussion/implications sections that your data are likely MNAR and therefore you cannot generalize beyond your sample.

AND

- Step 2a: Pick your poison - choose how you will handle your missing data

\section{AND/OR}

- Step 2b: Preregister your missing data decisions (optional but strongly recommended) 


\section{Step 2: Pick your Poison - Decide which Method to Use}

- Determining how to handle missingness is done by analysis.

- The decision about how to handle missing data is not a dataset-specific decision. Instead, it's a decision you should make for each analysis, specific to a given hypothesis.

\section{- Option A: Deletion Methods}

- Use this option when you decide NOT to adjust for missing data. There are two deletion methods that are relevant:

\section{$\circ$ 1. Listwise Deletion}

- Also called complete case analysis, listwise deletion occurs when all data from a case (e.g., a person or an item) is dropped from analyses because that case has missing data on at least one of the variables of interest (e.g., missing an outcome or a predictor). This is applied across all analyses.

- Generally, listwise deletion assumes MCAR. We do not recommend listwise deletion for data that are MAR or MNAR.

- Under MAR, listwise deletion has a high risk of introducing selection bias into your inferences.

- For example, imagine you are interested in examining the link between education and gender. These constructs may each relate to the extent to which participants have missing data. If so, using listwise deletion will introduce a spurious association (or spuriously modify the association) between education and gender.

- How this dynamic leads to bias can be formally shown using causal diagrams (e.g., Hernán et al., 2004)

- Some researchers prefer to use deletion when their data are MNAR because the information contained in other variables 
is not likely to increase precision or reduce bias in estimates.

At the very least, if you do use deletion, you should discuss why you think your data are MNAR and that for this reason you cannot generalize beyond your sample.

- In contrast, with MCAR data, using deletion will not introduce bias in your inferences. This is because the sample on which you have complete data is essentially a simple random subsample of your larger sample.

- If you have a large dataset, using more complex methods (e.g., multiple imputation) can take a lot of computational overhead. If there is a low proportion of missingness $(<5 \%)$ and your data are MCAR, bias introduced by listwise deletion is inconsequential, and this method can save time (Schafer, 1999).

- You should also consider the ethical implications of dropping cases. For example, participants are often completing a study with the intention that their data will contribute to the research. Dropping a person's responses can conflict with participants' intentions and expectations of how their data may be used.

- 2. Pairwise Deletion

- Pairwise deletion occurs when the statistical procedure uses complete cases on an analysis-by-analysis basis. For example, when computing a correlation table between a set of variables, computing a correlation for the first pair of variables will only use cases with data on both variables. Likewise, computing a correlation between the second pair will only use cases with data on both of those variables. This is repeated for each analysis (e.g., correlation) completed.

- Pairwise deletion typically assumes MCAR. The pros and cons of pairwise deletion for MCAR data are similar to listwise deletion. Although pairwise deletion tends to retain more cases than listwise deletion, deletion in general can be inefficient and wasteful. If there are ways to use data from cases with missingness (e.g., multiple 
imputation), you will have lower power to run your analyses if you instead rely on deletion (i.e., you simply lose cases in your sample).

- We also do not recommend pairwise deletion for MAR or MNAR data because there are more powerful and unbiased options available. Though you may be dropping fewer cases than with listwise deletion, you are still systematically removing cases and omitting covariance data from informing your final model.

- Moreover, to the extent that missigness on one of the two pairwise-deleted variables (e.g., drinking and marijuana use) is related to a third variable (e.g., income) and is therefore MAR, you may also be reporting a biased estimate because you systematically remove cases by levels of a third variable.

\section{- Option B: Multiple Imputation}

- Multiple imputation allows for uncertainty about the missing data by creating several different plausible imputed datasets and appropriately combining results obtained from each of them. When done correctly, this process yields consistent, asymptotically efficient, and asymptotically normal estimates (Allison, 2012).

- There are three basic steps to multiple imputation (from Allison, 2012):

- 1) Introduce random variation into the process of imputing missing values, and generate several datasets, each with slightly different imputed values.

- This is by far the most complicated step, and where most of your decisions about multiple imputation will be made.

- 2) Perform an analysis on each of the datasets.

- 3) Combine the results from analysis into a single set of parameter estimates, standard errors, and test statistics.

- When your data are MCAR or MAR...

- Pros to multiple imputation:

- Regain data points and increase power 
- With MCAR, you do not need to include auxiliary variables in your model, which increases speed and decreases complexity

- Cons to multiple imputation:

- Potentially computationally- and time-demanding, so it may not be worth it if the sample size is large and the number of missing cases are low

- When your data are MNAR...

- You may conduct multiple imputation if you wish to retain power and minimize any MAR bias that exists in your dataset. However, you should be aware that no matter what you do, you will still experience a loss of generalizability. You should therefore discuss why and how your sample is biased or non-generalizable due to MNAR data.

\section{- Option C: Full Information Maximum Likelihood (FIML)}

- FIML estimates the value of some population parameter by determining the value that maximizes the likelihood function based on all the observed variables for each case (Cham et al., 2017; Liu \& Sriutaisuki, 2021). It does so by choosing a parameter value that assigns the highest possible probability or probability density to the data values actually seen (Collins et al., 2001).

- When your data are MCAR or MAR...

- Pros to FIML (same as multiple imputation):

- Regain data points and increase power

- With MCAR, you do not need to include auxiliary variables in your model, which increases speed and decreases complexity

- Cons to FIML:

- Potentially computationally- and time-demanding, so FIML may not be worth it if the sample size is large and the number of missing cases are low 
- You also have a bit less control over the FIML algorithm relative to multiple imputation. It's a bit like a black box, where you specify your analytical model and auxiliary variables and then FIML *does magic* and gives you a complete sample. In other words, be wary of not understanding or misunderstanding how the method is addressing missing data.

- For more on the distinctions between multiple imputation and FIML, see the FAQ.

- When your data are MNAR...

- Similar to multiple imputation, you may conduct FIML if you wish to retain power and minimize any MAR bias that exists in your dataset. But again, you should make sure to discuss why and how your sample is biased or non-generalizable due to MNAR data.

- I've picked my poison. What are my next steps?

- Step 1: Preregister your missing data decisions (optional but strongly recommended)

\section{AND}

- Step 2: Test for missingness mechanism 


\section{Step 2.5: Preregister your missing data decisions (optional but strongly recommended)}

- When do you preregister your missing data decisions?

- Ideally, you would preregister your missing data decisions before you touch your data. This may not always be practical, though, so remaining transparent about where you are in the process at the time of preregistration is crucial.

\section{- What is preregistration?}

- Pre-registration is the act of publishing the plan for a study, including research questions/hypotheses, research design, and data analysis before the data has been collected or examined (e.g. Mertens \& Krypotos, 2019; Nosek et al., 2018; Tierney et al., 2020, 2021). The more detailed a pre-registration, the better third parties can assess these changes and with the validity of the performed analyses.

- Those unfamiliar with preregistration may be concerned that any deviations from their original plans may undermine their entire project. This is not the case. In fact, a recent project, emerging from an Association for Psychological Science Hackathon, found that all papers published in Psychological Science between February 2015 and November 2017 with a preregistration badge had deviated in some way (Claesen et al., 2019). The key here is transparency. If your preregistered plans differ from the final analysis, you should clearly disclose this. 
Table 1. Guides and Templates for Pre-Registration

\begin{tabular}{ll}
\hline \multicolumn{1}{c}{ Method/Subfield } & \multicolumn{1}{c}{ Source } \\
\hline Clinical science & Benning et al. (2019) \\
Cognitive modeling application & Crüwell \& Evans (2020) \\
Developmental cognitive neuroscience & Flourney et al. (2020) \\
EEG/ERP & Paul et al. (2021) \\
Experience sampling & Kirtley et al. (2021) \\
Experimental social psychology & van 't Veer \& Giner-Sorolla (2016) \\
Exploratory research & Dirnagl (2020) \\
fMRI & Flannery (2020) \\
Infant research & Havron et al. (2020) \\
Intervention research & Moreau \& Wiebels (2021) \\
Linguistics & Roettger (2020) \\
& Mertzen et al. (2021) \\
Psychopathology & Krypotos et al. (2019) \\
Qualitative research & Haven \& Van Grootel (2019) \\
& Haven et al. (2020) \\
Quantitative research & Bosnjak et al. (2021) \\
Replication research & Brandt et al. (2014) \\
Secondary data analysis & Weston et al. (2019) \\
& Mertens \& Krypotos (2019) \\
Single-case design & Van den Akker et al. (2021) \\
Systematic review (general) & Johnson \& Cook (2019) \\
Systematic review and meta-analysis & Van den Akker et al. (2020) \\
protocols (PRISMA-P) & Moher et al. (2015) \\
Systematic review (non-interventional) & Shamseer et al. (2015) \\
\hline
\end{tabular}

Source: Corker, K. S. (2021). An Open Science Workflow for More Credible, Rigorous Research (preprint). DOI: 10.31234/osf.io/wu6sn 
- What should you preregister?

- Theory about why your data are missing at random/completely at random/not at random

- The method you plan to use to identify missingness (see $\underline{\text { Step 2) }}$

- The auxiliary variables you will test

- The effect size or decision rule you plan to apply to determine whether a variable should be included as an auxiliary variable in multiple imputation or FIML

- The multiple imputation model you have chosen (with the predictor matrix - both key predictors and auxiliary variables - where appropriate)

- An explanation of how the multiple imputation model is congruent with the analysis model

- Why does this matter?

- Pre-registration should be conducted in order to handle missing data and avoid any researcher "degrees of freedom" (i.e., individual decisions) that may lead to significant results, leading to poor reproducibility and replicability of psychological findings (e.g. Open Science Collaboration, 2015; Nosek et al., 2018).

- l've preregistered (yay!). What are my next steps?

- Test for missingness mechanism 


\section{Step 3: Test for the Missingness Mechanism}

- When should you start testing your data to confirm whether your missingness occurs (not) at random?

- After theorizing/preregistration, but before imputing

- Why evaluate patterns in missingness?

- Understanding the scope of missingness will make it more clear how the way you handle missingness has impacted your results and conclusions.

- We would also like to add a shameless plug for the importance of designing studies that will obtain high quality data and to avoid any avoidable missingness. For example, if you know that certain subgroups may be less likely to participate or return surveys, make sure you include these descriptors in your study design so that you do not end up with non-ignorable missingness. To quote Light and colleagues (1990), "you can't fix by analysis what you bungled by design."

- 1. Examine patterns of missingness - for entire scales, items within scales, patterns of missing data points, groups of people

- Univariate descriptive statistics

- Check for any illogical values that could be missing data codes

- Consider implications of different data codes (e.g., missing data for "I prefer not to answer", "not applicable", or because someone didn't provide an answer each have different implications)

- Multivariate descriptive statistics

- Look for outliers and try to determine whether the data are "real" responses or can be considered skip patterns (e.g., careless responders or straight-line responders; King et al. 2018)

- Look for missingness at many variables simultaneously to identify missing data patterns. For example, people might be missing all of the cognitive tasks but none of the survey data (some example visualizations) 
- 2. Evaluate how much missingness you have on each variable of interest Remember to pay attention to the percent of both item-missing data (i.e., skipped questions on a survey) and attrition-missing data (i.e., people that were missing data for the entire wave - sometimes indicated by skip codes within datasets).

0 3. Choose a decision rule for determining whether missingness is related to other variables in your dataset

- This decision rule would ideally be preregistered!

- In large datasets, $p$ values may not be a reliable method to determine which variables meaningfully predict missingness patterns (i.e., there may be statistically significant differences at $p<.05$ because analyses are highly powered, but these differences may not be practically or meaningfully important).

- Effect sizes are a perfectly valid way to determine whether something is related to the missingness.

- How big of an effect size is big enough to matter? It depends, of course, on what the outcome is you're interested in. (Again, this is where preregistration comes in handy.) Pick an effect size that is the smallest relevant effect (Sullivan \& Feinn, 2012).

- If you are struggling with what a "smallest relevant effect" might be, some suggest a general rule of $r>0.4$ correlation as a threshold for inclusion (e.g., Collins et al., 2001; Enders, 2010) as this value seems to find a good balance between model parsimony and increased statistical power. (Note: you may have a "smallest relevant effect" that is $r<0.4$ - that's okay, but make sure you document and justify why you expect this to be the case) (UCLA Statistical Consulting Group).

- 4. Check whether any auxiliary variables are related to your missingness

- Auxiliary variables are variables in your data set that are not of particular interest to your analysis, but that are either correlated with a missing variable(s) or are believed to be associated with missingness. Auxiliary 
variables will be added to the imputation model to increase power and/or to help make the assumption of MAR more plausible, because including these variables has been found to improve the quality of imputed values generated from multiple imputation. This is especially important when imputing a dependent variable and/or when you have variables with a high proportion of missing information (Johnson \& Young, 2011; Young \& Johnson, 2010; Enders, 2010).

- You should still check for potential auxiliary variables even if your data are MCAR or MNAR!

- With MCAR data, just double check to make sure that there is nothing else predicting patterns of missingness.

- With MNAR data, your sample will be biased no matter what. However, you can lessen any additional bias and increase your power by using $\mathrm{Ml}$ and including auxiliary variables that may explain other missingness patterns.

\section{- Test auxiliary variables for inclusion}

1) Create a new dummy variable that represents whether your key variable is missing (missdummy=1) or not missing (missdummy=0).

2) Then predict it with auxiliary variables you think are likely to be related to missingness (e.g., t-tests for continuous auxiliary variables, chi-square for binary auxiliary variables, anova for categorical auxiliary variables). For example, in many longitudinal academic datasets, variables like parent education, mobility, income to needs ratio, and disability status are often related to higher dropout (attrition) in longitudinal studies.

3) Repeat this for any key variables you plan to include in the analysis (predictors; outcomes).

- Note: Assume that any statistical MCAR tests are actually tests for whether your data can be listwise deleted (van Ginkel et al., 2020); also, see $\underline{F A Q}$ 
- I have evaluated my data. Now what do I do?

- 1. Create a table of which variables are or are not related to missingness on your key variables

- If you encounter page limitations that hinder your ability to include this table in your main manuscript, you can plan to include this with your supplementary materials. Supplementary materials can be very useful, particularly for journals with shorter page limits. Because most journals are disseminated digitally now, your article can include a hyperlink to your supplementary materials.

\section{- 2. Decide which auxiliary variables to include in missing data adjustment procedures and retain those variables}

- Once you've identified everything that you think could be *theoretically related to your missingness* and confirmed which are in fact related (i.e., you've got a good idea of who the data are missing for), then you can consider the data on your key variables to be MAR.

- Keep any variable that was significantly and/or meaningfully related to the missingness (based on your decision rules) in your imputation model along with your key variable(s). They're your auxiliary variables. They help you get less biased estimates of your relations.

- It is better to err on the side of more rather than fewer auxiliary variables, as there are minimal downsides to including many auxiliary variables in imputation procedures and their inclusion improves the likelihood of the MAR assumption holding (Enders. 2010).

- The only downside to including a large number of auxiliary variables could potentially be computation time or model complexity. In fact, White et al. (2011) note that including more than 70 mostly categorical variables or more than 100 
mostly continuous variables may pose convergence difficulties.

- If you encounter convergence issues for this reason, begin by eliminating the most tangential auxiliary variables. Anything that is significantly or meaningfully related to your key variable(s) MUST remain included in the imputation model, or your resulting dataset will be biased.

- Using all predictors may not be feasible or necessary in datasets that contain hundreds of variables (i.e., because you will likely encounter issues with multicollinearity and computational problems). van Buuren (2018) notes that the increase in explained variance in linear regression is typically negligible after the best $\sim 15$ variables have been included. For this reason, it is most expedient to select a suitable subset of data that contains no more than 15 to 25 variables (van Buuren, 2018)

- Researchers should also use the Fraction of Missing Information (FMI) to guide their choice of auxiliary variables for efficiency gain in imputation analyses (Madley-Dowd et al., 2019). 


\section{Step 4: Data Carpentry - Setting Up Your Data}

- When do you set up your data for multiple imputation?

- After testing for MAR in your dataset, but before imputing values

\section{- Considerations}

- Check your distributions

- The same assumptions that underlie any regression-based analysis also underlie multiple imputation. If you use a regression-based method to predict categorical or markedly non-normal imputed values, you may be setting yourself up for the imputation to fail or to introduce bias. Address violations of the assumption of normality prior to imputing, or make sure that your imputation method is appropriate for your distribution (e.g., classification and regression trees; Brieman et al., 1984).

- Fit the proposed regression imputation model to the observed data prior to multiple imputation, and perform regression diagnostics. If diagnostics suggest poor model fit, the imputation model could be altered prior to producing imputations.

\section{- Transform variables prior to imputation}

- For instance, if you know you will be using standardized variables in your analysis, standardize variables prior to imputation. The same goes for interaction terms or variable transformations (e.g., to correct skew/kurtosis).

- It is typically good to create any interaction variables between continuous variables that the researcher intends to investigate analytically prior to imputation (Allison, 2002). Enders et al. (2014) provides a guide (with some software syntax) for dealing with continuous $x$ continuous interactions.

- However, categorical variables might be imputed separately prior to calculating an interaction (Allison, 2002). 
- If you have scales, you would ideally create the scale first and then impute that scale score. This is known as the Just Another Variable, or JAV, approach.

- However, this might not be the best idea if the scale is missing quite a lot of data due to missingness on each individual item (e.g., if 1 of 7 items is missing, the entire scale score for that person will be missing).

- If this is the case, impute each item separately and then recreate the scale at the end. You can also impute into the (mostly missing) scale as well as into each item and compare results as a sensitivity check.

- This could be an important place to reevaluate for MAR/MNAR - for example, if all 7 items on a scale are missing for an individual, WHY might this be the case? Can you predict this attrition from other variables in your dataset?

- You might also be interested in relations between particular items in scales. In this case, item-level imputation would be best, rather than creating the scale first.

- There are other methods as well that may be better than JAV or imputation and then scale creation. One possibility is Passive Imputation. This method transforms variables on-the-fly within the imputation algorithm, thus hopefully removing the bias of the impute-then-transform method while restoring the consistency that was broken among the imputation in JAV (van Buuren, 2018).

- Impute into both your dependent variable(s) and your predictor(s) of interest

- While some researchers have cautioned against using the dependent variable in an imputation model, Graham (2009) calls this a "myth" because not including the dependent variable may produce biased estimates (i.e., why would you expect missing data to bias only your predictors and not your outcome variables?) 
- Auxiliary variables may be used to impute but do not need to be imputed themselves. In some software programs, auxiliary variables that are missing data themselves may need to be imputed to prevent imputation of missing values on key variables of interest.

\section{- Impute categorical data (or, not)}

- You should think critically about whether all categorical variables are reasonable to impute (e.g., demographic information). Is it defensible to use information on $X$ (for instance, a participant's level of neuroticism or extraversion) to predict $Y$ (for instance, gender)? (see $\underline{F A Q}$ )

- If you do decide to impute, categorical or ordinal data can be imputed using the same methods as you might find in an analysis (e.g., logistic regression, multinomial logistic regression, ordinal regression).

- Alternatively, predictive mean matching (PMM) uses a linear regression model to impute continuous missing data, a logistic regression model to impute binary missing data, and a multinomial logistic regression model to impute categorical missing data. However, instead of imputing random values from the conditional distribution, these regression models are used to find observed cases that most closely resemble the predicted values of the respondents with missing data. In this way, multiple imputation using PMM results in imputed values that are actually observed in the dataset and that are more robust to violations of normality than other approaches (i.e., regress, logit, mlogit) (van Ginkel et al.. 2020).

\section{- Do not impute data that should be missing}

- This may depend on your data, but in some cases it makes sense not to impute data that are missing due to skip logic or assignment to condition. 


\section{Include weights when appropriate}

- Manly and Wells (2015) recommend that weights be included in imputation commands when software allows, and complex survey design elements such as the primary sampling unit should be included in the imputation model as predictors (Heeringa et al. 2010; Azur et al., 2011).

- The imputation model is actually weighted in the same way an analysis would be weighted (i.e., in Stata you can include the term [pweight=weight] in the same place in your imputation model as you would in your analytic model)

\section{- Nested data considerations}

- If you have nested data, you also should account for this by setting up a multilevel imputation model.

- There are multiple ways to handle multi-level data (also see below re: congeniality)

- Manly and Wells (2015) provide a good summary of a multilevel imputed model, and their Stata code is included in our Stata syntax template on our OSF page (https://osf.io/j3f8m/)

\section{- The importance of congeniality}

- The imputation model and the analytic model must match.

- As a general rule, whatever is included in the analytic model needs to be included in the imputation model (e.g., interaction terms; random intercepts, random slopes, and contextual effects in multilevel models)

\section{○ How long will the imputation take?}

- Approximating imputation time can be helpful and will depend on both how complex your model is (e.g., the number of variables and the types of chained equations models you are using for different variables) as well as your computer's processing speed. 
- For example, Treiman (2009) found that 'approximately doubling the number of variables to be imputed increased the time [to impute] by a factor of four' (p. 186).

- White and colleagues (2011) note that including more than 70 mostly categorical variables or more than 100 mostly continuous variables may pose convergence difficulty.

- Certain types of chained equations models used for different variables also take more time. For instance, multinomial logistic regression takes noticeably longer than OLS or logistic regression (White et al. 2011). This is one reason why using a method like predictive mean matching can be beneficial.

- Manly and Wells (2015) note that for chained equations, imputation time can be estimated by multiplying the time for a single chain by both the number of burn-in iterations and imputations. They suggest using 2 imputations ( $m=2$ ) for speed when initially making model adjustments before increasing to a larger $\mathrm{m}$ for final models.

- My data are set up and ready to go! What are my next steps?

- Multiply impute your data 


\section{Step 5: Multiply Impute Your Data}

- Choose the type of multiple imputation model

- Multivariate Normal Imputation (MVN)

- Also known as data augmentation (DA)

- Starting values for missing data are computed given the current regression parameters and observed data, and this "augmented" data is used to compute new parameters.

- Only use this if you have all continuous variables

- This is only recommended for research solely involving continuous variables because MVN's assumption of joint multivariate normality does not hold for categorical variables.

- See Allison, 2002; Enders, 2010; Graham 2009; McKnight et al. $\underline{2007}$

- Markov chain Monte Carlo (MCMC)

- Bayesian approach

- Based on the Bayesian paradigm, MCMC can be used for an arbitrary missing data pattern that assumes multivariate normality (Schafer, 1997). A Markov chain is a sequence of random states (or samples) of the variable at a given point in time in which the distribution of each element depends on the value of the previous.

- Use this for small samples that are MAR with multivariate normality

- The advantage of MCMC is that there is no need to rely on large sample assumptions because exact posterior distributions can be estimated for any distribution of the model unknowns. In small to moderate samples, these posteriors can provide a more realistic measure of model uncertainty than frequentist approaches.

- Key assumptions are that data are missing at random, and that all of the variables with missing data have a multivariate 
normal distribution (an implicit assumption of linear regression imputation). This latter assumption may lead to implausible imputed values in some analyses.

- MCMC imputation begins with reasonable starting values for the means, variances, and covariances among the variables (i.e., through listwise or pairwise deletion). Then the sample is divided into subsamples, each with the same missing data pattern (i.e., the same set of variables present vs. missing). For each pattern, the starting values are used to construct linear regressions for imputing the missing data using all the observed variables in the pattern as predictors. The missing values are then imputed through random draws from the simulated error distribution, which results in a single "completed" dataset that is in turn used to recalculate the means, variances, and covariances. A random draw is then made from the posterior distribution of these recalculated parameters, which is used to update the linear regression equations needed for imputation. This process then repeats many times (iterations) (Allison, 2012).

- See Allison, 2000, 2012; Baraldi \& Enders, 2010; Croy \& Novins, 2005; Kenward \& Carpenter, 2007; Lee \& Song. 2004; Peugh \& Enders, 2004; Taylor \& Zhou, 2009

\section{- Chained (MICE/ICE)}

- Also known as sequential regression imputation, or fully conditional specification (FCS)

- This is the default method, and is what *most people* will use

- MICE has empirically been shown to produce good results for data with a mixture of continuous and categorical variables (van Buuren et al. 2006; van Buuren, 2007).

- Once an initial iteration through the data provides starting values for all missing data, each variable is evaluated using the specified model (e.g. logistic, predictive mean matching, 
regression, etc.) to impute values for missing data using imputed values from the previous iteration.

- However, this is the slowest method computationally.

- See Allison, 2012; Royston \& White, 2011; Social Science Computing Cooperative, 2012; van Buuren, 2018; van Buuren et al. 2006; van Buuren \& Groothuis-Oudshoorn, 2011

- Note: Classification and regression trees (CART) fall under the MICE approach. CART is a nonparametric approach to handling missing data that employs machine learning algorithms. It may be useful for large epidemiological samples that include outliers, skewed data and multicollinearity (Breiman et al., 1984; van Buuren, 2018).

- Set up your MI model

- Make it reproducible

- Some software programs allow you to set a seed number so you can reproduce the imputation if necessary (e.g., 12345)

- Note: if you preregister your missing data method, include this seed code as well!

- Match to your planned analyses

- Weights

- If you plan on using weights in your analyses, you should include these in the imputation model

- Model specifications

- If you are planning a multilevel model, you should specify a multilevel imputation procedure

- $\mathrm{HMI}$ and mice currently allow 2-level imputation models in $\mathrm{R}$

- Choose the number of imputations $(m)$

- There are several recommendations currently available for how to decide $m$. 
- Previous researchers have suggested that five datasets may be adequate for most analyses (Allison, 2002; Schafer \& Olsen, 1998), while others recommend 2-10 imputations suffice (Rubin, 1987). We do not advise these recommendations because they are focused on maximizing efficiency of point estimates until a desired result, such as $p<.05$, is obtained. This leads to non-replicable results, reducing the openness and transparency of scientific research (Freese, 2007).

- von Hippel (2018) recommends a two-stage procedure. First, conduct a pilot analysis using a small-to-moderate number of imputations. Second, use results from the pilot analysis to calculate the number of imputations for a final analysis to allow standard error estimates to reach the recommended level of reproducibility and replicability.

- Yet, again, this procedure runs the risk of impairing replicability because researchers may be tempted to select a number of imputations $m$ that maximize efficiency of a desired result, such as $p<.05$, which can lead to non-replicable results (Freese, 2007).

- Graham and colleagues (2007) suggest that the number of imputed datasets should increase as the amount of missing data increases. White et al. (2011) also recommend a rule of thumb that $m$ should be at least equal to the highest percentage of incomplete cases (i.e., missing data occurs for $30 \%$ of cases $\rightarrow m>=30$ ).

- However, for an adequate level of reproducibility in practice, several others recommend against using the proportion of missing data to guide decisions, and instead suggest using the Fraction of Missing Information (FMI) (Madley-Dowd et al., 2019; White et al., 2011).

- The FMI is the fraction of between-imputations variance divided by the average within-imputation variance

- $\mathrm{FMI}=\mathrm{B} /(\mathrm{W}+\mathrm{B})$, where 
- $\mathrm{B}=$ between-imputations variance; an estimate of the additional sampling variability produced by the imputation process (Allison, 2012)

- $\mathrm{W}=$ Average within-imputation variance; the mean of the squared standard errors from the separate analysis of the several datasets (Allison, 2012).

- The interpretation of FMI is similar to an R-squared. So an FMI of 0.15 means that $15 \%$ of the total sampling variance for that variable is attributable to missing data.

- Under these recommendations, the number of imputations $m$ should be chosen so that dividing the FMI by $m$ should be less than or equal to about $\sim 0.01$ (in other words, signaling roughly a loss of precision of $1 \%$ or less; White et al., 2011).

- But, the tricky thing about the FMI is that it changes based on $m$. In other words, the FMI is unreliably estimated unless $m$ is rather large (Graham et al., 2007).

- The larger the amount of missing information...

- 1) the higher the FMI needed to reach good relative efficiency,

- 2) the higher the chance you will run into estimation problems during the imputation process, and

- 3) the lower the chance of meeting the MAR assumption unless it was planned missing (Johnson \& Young. 2011; UCLA Statistical Consulting Group).

- Some have even recommended that you increase the number of imputations quadratically with the fraction of missing information so as to not change standard error estimates during re-imputation (von Hippel, 2016). 
- A relatively safe approach might be to impute at least the highest percentage of incomplete cases, and then check to make sure that the $\mathrm{FMI} / m<=0.01$.

\section{- Make sure your imputation model worked!}

- Double-check precision

- $\mathrm{FMI} / m<=0.01$, or $1 \%$

\section{- Check for proper convergence}

- For chained equations, where there is no theoretical proof of convergence, van Buuren (2018) discusses and illustrates differences between reasonable and problematic convergence (e.g. see his example plots on in section 6.5.2).

- The autocorrelation plot is very useful for assessing convergence. Autocorrelation measures the correlation between predicted values at each iteration. Because the imputation process is designed to be random, we should not observe correlated imputed values across imputations. So, if you noticed a trend in the variances in the previous trace plot, you may want to assess the magnitude of the observed dependency of values across iterations through an autocorrelation plot.

- Investigate trace plots

- 1. Examine the plot to see if the predicted values remain relatively constant and that there appears to be an absence of any sort of trend (called a "stable posterior distribution"). This means the values jump kind of randomly around the center threshold, and imply a sufficient amount of randomness in the coefficients, covariances, and/or variances between iterations.

- 2. Examine the plot to see how long it takes to reach this stationary or stable phase. Good convergence is implied when this looks like it happens almost immediately (i.e., no wandering-off values, no observable pattern). If proper convergence is not achieved, you can increase the burn-in 
period, or the number of iterations before the first set of imputed values is drawn.

- Because convergence takes time, the burn-in (or warmup) iterations help the sampler explore the probability space more effectively.

- The purpose of burn-in is to let the chain "settle" in the probability space, so that it gives us better samples once we actually start recording the samples. The reason for this is that the initial states of the chain depend a lot on the values that were used to initialize the chain. Letting the chain "burn-in" (warm up) lets it settle in the part of the probability space we want to be exploring.

- See: Enders, 2010; Rubin, 1987

- https://rdrr.io/cran/mice/man/plot.mids.html

- https://support.sas.com/documentation/cdl/en/statug/6 7523/HTML/default/viewer.htm\#statug_introbayes_se ct024.htm

- https://stats.idre.ucla.edu/stata/seminars/mi in stata pt1 new/

\section{- Look at descriptive information}

- Look at your means and frequencies, and compare observed and imputed values to assess if the range appears reasonable or plausible. In other words, do your imputation results make sense?

- Sometimes it's helpful to evaluate this graphically. You can do this through histograms, boxplots, density plots, cumulative distribution plots, strip plots, and quantile-quantile plots with the observed and imputed data (see section 6.5.2 in van Buuren, 2018).

- Plot observed data points versus residual data points.

- After performing multiple imputation, residuals can be generated for each completed dataset. 
- For individuals with observed data, the residual = observed value minus the prediction from the analysis model

- The imputed data residual = imputed value minus the prediction from analysis model

- If data are missing at random, observed and imputed distributions should look similar with regard to response probability.

- For example, you can estimate probabilities of response using a logistic regression model with the missing data indicator as the outcome variable and completed variables as predictors separately for each imputed dataset.

- Check imputations graphically by plotting observed scores against estimated response, perhaps using different colored markers for observed and imputed data.

- Check the goodness-of-fit of the imputation models

- Use established goodness-of-fit models to check assumptions of regression models. Standard regression diagnostics include investigations of residuals, outliers, and influential cases.

- You may also want to examine plots of residuals and outliers for each imputed dataset individually. If anomalies are evident in only a small number of imputations then this indicates a problem with the imputation model (White et al, 2011).

\section{- Assess the predictive ability of your model}

- Leave-one out cross-validation can be used to assess the predictive ability of a model. A single observation is deleted and the proposed model is fitted to the remaining data and used to predict the outcome for the excluded data point. This is repeated by cycling through each observation, deleting and predicting the outcome for each observation in turn. The model can graphically assess by plotting predicted values against observed values (Gelman et al.. 1998)

- Posterior predictive checking (PPC) creates replicated datasets from the proposed imputation model (Gelman et al., 2013). You 
then compare inferences from imputed data to inferences from replicated data. Here, you're looking at the potential effects of model inadequacies on results of interest. Some have argued that this is more informative than focusing on the quality of imputed data values by comparing imputed vs. observed values (Gelman et al., 2005; Gelman et al., 2013; He \& Zaslavsky, 2011; Nguyen et al., 2015).

- Note that you probably also want to compare the results from your imputed data to results from complete (listwise deleted) data. It can be important to report how much your inferences and estimates might differ for listwise deletion vs multiple imputation; this can show the amount of bias you might have avoided by adjusting for missing data (assuming that listwise is always going to introduce more bias).

- I have imputed my data and checked that everything went smoothly. Now what do I do?

- Report your Results 


\section{Step 6: Report your Results}

- You did it! You're almost there - now you just have to report what you did, how, and why.

- Amount (rates) of missing data

- Descriptive statistics before/after multiple imputation, including \% missing data for each variable (ideally, this would also include auxiliary variables, but these can go in a supplement if necessary)

- Use formal numerical methods to compare distributions of observed and imputed values to highlight variables that are of concern (Stuart et al., 2009). Flag any variables with ratio of variance between imputed and observed values being less than 0.5 and higher than 2 or if any absolute differences in mean is greater than 2 standard deviations. This may be evidence that your imputation model was misspecified unless you have good reason to expect the values to change this dramatically (and justify this in your paper).

- Report information about missingness mechanism and why you think this is the case

- MCAR if data were randomly assigned to missing conditions or missingness was caused by technical errors or issues, AND there are no other auxiliary variables related to missingness in your dataset

- MAR

- Possible variables or scenarios that could explain missing data

- Results of diagnostic tests (i.e., evidence of Ignorable Patterns or Assumptions; Manly \& Wells. 2015)

- You MUST REPORT variables that were retained as auxiliary variables in imputation models

- MNAR if you can think of reasons why you can't generalize beyond your sample

- Report the multiple imputation model setup

- Specify the basic software command and any key non-default options 
- Software (R, Stata, SAS, etc.) and its version number

- Type of multiple imputation (i.e., chained, hmi, Amelia, etc.)

- Seed number for reproducibility

- How many $m$ datasets and how you arrived at that decision

- Pooling procedures (typically "Rubin's rules" [1987] wherein parameter standard errors are combined incorporating both the within-imputation variance and the between-imputation variance)

\section{- Report results from your checks to make sure the imputation worked}

- Report precision (FMI/m)

- Report convergence and the number of burn-in iterations, including autocorrelation or trace plots

- Report descriptive comparisons of observed vs. imputed values, including the plots you investigated to compare observed and imputed data

- Report results from posterior predictive checking (PPC), as well as results from listwise deletion, and discuss how your imputed results differ from what you might have obtained had you not adjusted for missing data bias

\section{- Example methods paragraphs}

- Enders (2010):

- Prior to performing the analyses, we used multiple imputation to deal with the missing data. Briefly, multiple imputation uses a regression-based procedure to generate multiple copies of the data set, each of which contains different estimates of the missing values. We used the data augmentation algorithm in the SAS MI procedure to generate 50 imputed data sets (Graham et al., 2007, recommend at least 20 for most situations). Graphical diagnostics from an exploratory analysis suggested that the data sets should be separated by at least 50 iterations of the imputation algorithm, so we took the conservative tack of saving the first data set at the 300 th iteration and saved additional data sets every 300 th iteration thereafter. The imputation process included the 13 variables that appeared in one or more of the subsequent regression analyses as 
well as three auxiliary variables (body mass index, peer pressure, and parental ratings of food preoccupation). After creating the complete data sets, we estimated the multiple regression models on each filled-in data set and subsequently used Rubin's (1987) formulas to combine the parameter estimates and standard errors into a single set of results. Note that methodologists currently regard multiple imputation as a "state of the art" missing data technique because it improves the accuracy and the power of the analysis relative to other missing data handling methods (Schafer \& Graham, 2002).

- Manly \& Wells (2015):

- The percentage of missing values ranged from nearly 0 for some demographic variables to as high as $12 \%$ for data about student loans, and only $88 \%$ of the 14,015 students in the sample would have been available for analysis under the traditional listwise deletion method. Data are primarily missing due to item nonresponse, and while participant attrition also exists, NCES refreshed the sample to keep the senior cohort data nationally representative. We address the problem of missing data using the "state of the art" [multiple imputation (MI)] technique including all analysis variables under the assumption that missing values are missing at random (Schafer \& Graham 2002). MI was used instead of NCES' single imputations for gender and race/ethnicity. Due to the complex design employed in the data collection, the primary sampling unit, strata, and weights were included in the imputation process (Heeringa et al. 2010). Stata 13's 'mi impute chained' command generated 20 imputed datasets, and visual inspection of imputation convergence led to the very conservative choice of 15 burn-in iterations (van Buuren 2012). Analyses run on each dataset were pooled according to Rubin's (1987) rules. Imputed values 
compare reasonably to observed values, and results using listwise deletion are similar to $\mathrm{Ml}$, so imputed results are presented. 


\section{Frequently Asked Questions}

- What about Little's MCAR test, can't I use that to determine if my data are MCAR?

- No. This is more of a test of whether you can use listwise deletion for your data (and just because you can listwise delete doesn't mean you should see Option A: Deletion Methods). Remember we can never prove that data are MCAR (unless you plan them that way on purpose).

- Little's MCAR test is generally not helpful because MCAR is not knowable from the data alone. It may give a false sense of security. With large datasets, it is also likely to be statistically significant (indicating violation of MCAR) because it's overpowered. For these reasons, we do not recommend this test.

- Similarly, the only thing that such null hypothesis significance testing tells us is that, when significant, the null hypothesis of the missing data being randomly scattered across the data has been rejected. Assuming, for the sake of argument, that no Type I error has been made, this rejection implies either MAR or MNAR.

\section{- Am I making up data or manufacturing results?}

- No! Especially not if you are comfortable with the logic of using regression analyses to estimate outcome values given a set of predictors. The logic of multiple imputation is similar -- given a set of predictors, you are estimating what those missing values are most likely to be.

- I did my analysis once, and then I imputed my data and my significant result went away! Doesn't that mean I shouldn't use imputation?

- No. If you only did a single imputation then this may be due to variance between imputations. This is why we recommend you do multiple imputations instead of just one single imputation.

- If you did use multiple imputation and still found differences between the listwise deleted result and the imputed result, that is not unexpected. 
Remember, every missing data decision you make comes with consequences for whom the results hold. If you have a significant relation with a listwise-deleted (i.e., complete case) dataset and not for one where you used multiple imputation, that tells you the results of your first analysis are only valid for a sample of people who have complete data. They do not hold for the entire sample.

- As another reframe, think of this like a robustness check. If your values change given a new specification of your model, your model might not have high validity, and you should spruce it up.

- For example, your results might have changed because your sample size and therefore the amount of information and variance have increased. If your significant results disappear after you add more observations to your sample, this may be due to the additional variance for those new observations.

- But can I impute every variable? Or, are there any variables we should not impute?

- It depends. For demographic variables like race and gender, you should think critically about the implications of using data on, say, personality and drug use, to make assumptions about somebody's personal characteristics.

- Are deletion methods and multiple imputation or FIML the only options?

- Single imputation and regression imputation methods are other options besides deletion and are described below for transparency. However, these methods are not as effective as multiple imputation or FIML. For this reason, if you choose to adjust for missing data (i.e., and therefore choose not to use a deletion method), we recommend that you use multiple imputation or FIML.

- Single Imputation Methods

- Single imputation methods are procedures that replace each missing observation with a single estimated value, often with the 
purpose of obtaining a "complete" dataset to be used for analyses (Fielding et al., 2008). Various methods can be implemented to impute missing data, each of which make their own sets of assumptions:

\section{- Variable Mean/Median Imputation}

- Fills in the variable mean/median for each missing value. This is the easiest single imputation method, but it will bias variances/covariances downward

\section{- Last Observation Carried Forward}

- In longitudinal data, when one time point is missing, you fill in with value from the last response. This is similar to variable mean imputation but does not bias variances as much (because the carried-forward value can be time-varying as opposed to one time-invariant mean). However, the variances will still be biased downward, and you will also assume little to no change in your construct over time which may not be appropriate.

- Single imputation methods require much less computational time and effort than more complex methods such as multiple imputation. This is particularly true when subsequent analyses are complex (e.g., a hierarchical structural equation model with a complex latent measurement model)

- Yet, when analyses are conducted on a dataset prepared using single imputation, these will treat the imputed values as "real" (without distinguishing them from responses that have actually been observed).

- In other words, single imputation does not take into account that there is uncertainty in the imputation, which can lead to potentially artificially small error terms, inaccurate and overconfident inferences.

- When your data are MCAR... 
- Single imputation will retain more cases than deletion methods, but will bias variances and covariances downward (i.e., because there are more values at the mean). This is less of a concern with MCAR data since missing values are a simple random subsample of all cases.

- When your data are MAR or MNAR...

- We do not recommend single imputation methods unless there is either (1) a strong theoretical reason to justify one particular type of single imputation, or (2) there are important practical concerns that make it infeasible to use modern methods (e.g., multiple imputation, FIML).

- For example, some have argued that single imputation methods are appropriate in specific domains when it is common for the missing data to have a predictable value, or in cases where very slow change over time occurs (i.e., researchers may assume that the last non-missing observation is almost always correct for more time-invariant phenomena like smoking status or family sociodemographic status. However, these are obviously debatable positions to take).

- Simple imputation methods have been found to be especially inadequate for missing not at random (MNAR) data (Fielding et al., 2008)

\section{- Regression Imputation Methods}

- Many similar pros and cons apply to regression imputation methods and single imputation methods. Although regression imputation methods are generally considered "better" than single imputation, they are still not perfect. For example:

- Regression Imputation 
- Fills in predicted values from a single regression model with other variables as predictors. This tends to work better than variable mean/median imputation, but will still bias variances downward and covariances upward (van Ginkel et al., 2020).

\section{- Stochastic Regression Imputation}

- Imputes a predicted value from a regression model plus a random error term. This is considered a better approach than regression imputation. If parameter bias were the only issue, this would be sufficient (although standard error estimates would still be too low). However, even this method will bias both $p$ values and widths of confidence intervals downward (van Ginkel et al., 2020).

- When your data are MCAR...

- Regression imputation will retain more cases than deletion methods and will result in "better" estimates than single imputation methods.

- When your data are MAR or MNAR...

- Estimates obtained under regression imputation methods will be more biased than estimates obtained under multiple imputation or FIML, because these methods do not account for random variation in imputation. In other words, the newly imputed values are treated as "real" instead of as estimates based on the conditional probability of your predictor variables. For this reason, we recommend multiple imputation or FIML over regression imputation methods. 
Table 2. Summary and Errors of Common Missing Data Techniques.

\begin{tabular}{|c|c|c|c|}
\hline & \multicolumn{3}{|c|}{ Missingness Mechanisms } \\
\hline & $\begin{array}{c}\text { Missing } \\
\text { completely at } \\
\text { random (MCAR) }\end{array}$ & $\begin{array}{l}\text { Missing at random } \\
\text { (MAR) }\end{array}$ & $\begin{array}{l}\text { Missing not at } \\
\text { random (MNAR) }\end{array}$ \\
\hline Definition: & $\begin{array}{l}\text { Random processes } \\
\text { lead to equal } \\
\text { probabilities of any } \\
\text { data point to be } \\
\text { missing. }\end{array}$ & $\begin{array}{l}\text { A variable value is } \\
\text { missing partly } \\
\text { depending on other } \\
\text { data observed in } \\
\text { dataset but do not } \\
\text { depend on any } \\
\text { missing value }\end{array}$ & $\begin{array}{l}\text { Probability a value is } \\
\text { missing depends on } \\
\text { the missing data } \\
\text { values themselves. }\end{array}$ \\
\hline Listwise Deletion & $\begin{array}{l}\text { Pros: Unbiased } \\
\text { results } \\
\text { Cons: Wasteful, } \\
\text { dropping usable } \\
\text { cases and low power }\end{array}$ & $\begin{array}{l}\text { Pros: None. } \\
\text { Cons: Biased results, } \\
\text { low power, cannot } \\
\text { generalise beyond the } \\
\text { sample. Do not use! }\end{array}$ & $\begin{array}{l}\text { Pros: None. } \\
\text { Cons: Biased results, } \\
\text { low power, cannot } \\
\text { generalise beyond the } \\
\text { sample. Do not use! }\end{array}$ \\
\hline Pairwise deletion & $\begin{array}{l}\text { Pros: Unbiased and } \\
\text { retain more cases } \\
\text { than listwise deletion } \\
\text { Cons: Wasteful, } \\
\text { dropping usable } \\
\text { cases and low power }\end{array}$ & $\begin{array}{l}\text { Pros: Unbiased and } \\
\text { retain more cases than } \\
\text { listwise deletion } \\
\text { Cons: Biased results, } \\
\text { low power, cannot } \\
\text { generalise beyond the } \\
\text { sample. Do not use! }\end{array}$ & $\begin{array}{l}\text { Pros: Unbiased and } \\
\text { retain more cases than } \\
\text { listwise deletion } \\
\text { Cons: Biased results, } \\
\text { low power, cannot } \\
\text { generalise beyond the } \\
\text { sample. Do not use! }\end{array}$ \\
\hline Single imputation & $\begin{array}{l}\text { Pros: Unbiased and } \\
\text { retains more cases } \\
\text { than listwise deletion } \\
\text { Cons: Inaccurate } \\
\text { standard errors, } \\
\text { variability reduced }\end{array}$ & $\begin{array}{l}\text { Pros: Less biased than } \\
\text { deletion methods } \\
\text { Cons: Often biased; } \\
\text { inaccurate standard } \\
\text { errors }\end{array}$ & $\begin{array}{l}\text { Pros: Less biased, but } \\
\text { still non-generalizable; } \\
\text { discuss why and } \\
\text { how the sample is } \\
\text { biased. } \\
\text { Cons: Inaccurate } \\
\text { standard errors, still } \\
\text { will not remove all bias }\end{array}$ \\
\hline $\begin{array}{l}\text { Regression } \\
\text { Imputation }\end{array}$ & $\begin{array}{l}\text { Pros: Unbiased and } \\
\text { better than single } \\
\text { imputation, retains } \\
\text { more cases than } \\
\text { deletion } \\
\text { Cons: Estimates } \\
\text { treated as "real" } \\
\text { instead of predicted }\end{array}$ & $\begin{array}{l}\text { Pros: Less biased than } \\
\text { single imputation } \\
\text { methods } \\
\text { Cons: More biased } \\
\text { than MI or FIML }\end{array}$ & $\begin{array}{l}\text { Pros: Less biased than } \\
\text { single imputation } \\
\text { methods, but still } \\
\text { non-generalizable; } \\
\text { discuss why and } \\
\text { how the sample is } \\
\text { biased. } \\
\text { Cons: More biased } \\
\text { than Ml or FIML, still } \\
\text { will not remove all bias }\end{array}$ \\
\hline
\end{tabular}




\begin{tabular}{|c|c|c|c|}
\hline $\begin{array}{l}\text { Full Information } \\
\text { Maximum } \\
\text { likelihood (FIML) } \\
\text { or Multiple } \\
\text { Imputation (MI) }\end{array}$ & $\begin{array}{l}\text { Pros: Unbiased; } \\
\text { accurate standard } \\
\text { errors } \\
\text { Cons: } \\
\text { Computationally- and } \\
\text { time-demanding, so } \\
\text { not worth it if sample } \\
\text { size is large }\end{array}$ & $\begin{array}{l}\text { Pros: Unbiased; } \\
\text { accurate standard } \\
\text { errors } \\
\text { Cons: } \\
\text { Computationally- and } \\
\text { time-demanding }\end{array}$ & $\begin{array}{l}\text { Pros: Least biased, but } \\
\text { still non- generalizable; } \\
\text { discuss why and } \\
\text { how the sample is } \\
\text { biased. } \\
\text { Cons: } \\
\text { Computationally- and } \\
\text { time-demanding, still } \\
\text { will not remove all bias }\end{array}$ \\
\hline
\end{tabular}

Source: Adapted from Newman, D. A. (2014). Missing data: Five practical guidelines.

Organizational Research Methods, 17(4), 372-411. https://doi.org/10.1177/ 


\section{- Where do I upload my preregistration?}

- There are many platforms for hosting preregistration. From Nosek et al., (2018), p. 2605:

- The World Health Organization maintains a list of registries by nation or region (www.who.int/ictrp/network/primary/en/), such as the largest existing registry, https://clinicaltrials.gov/.

- The AEA RCT Registry, the American Economic Association's registry for randomized controlled trials (https://www.socialscienceregistry.org), the Registry for International Development Impact Evaluations (RIDIE) Registry (https://ridie.3ieimpact.org/), and the Evidence in Governance and Politics (EGAP) Registry (https://egap.org/content/registration) are registries for economics and political science research.

- The Open Science Framework (OSF) (https://osf.io) is a domain-general registry service that provides multiple formats for preregistration (https://osf.io/registries/), including the flexible and relatively comprehensive Preregistration Challenge format (https://osf.io/prereg/).

- Finally, the website https://aspredicted.org/ provides a simple form for preregistration, but it is not itself a registry because users can keep their completed forms private forever and selectively report preregistrations. However, researchers can post completed forms to a registry to meet the preservation and transparency standards.

\section{- How much missingness is too much?}

- Many people are concerned about this question, but it is actually not the way to approach the issue of missing data in your data set. The real questions are: What decisions will you make about the data that are missing, and how will that missingness be handled? The missing data mechanisms and the missing data patterns have greater impact on research results than does the proportion of missing data (Tabachnick \& Fidell, 2012). For some situations you may choose to delete an entire 
participant's data because the frequency of response is near zero. There may be ethical reasons for not imputing into discrete categories such as sex, gender, race, immigration status categories and so you choose pairwise or listwise deletion of the data instead of imputation. Like many other data management decisions, the choices you make on what to do about missing data should be explicit and justified in your manuscript. Using logical reasoning and citing studies that have shown biases for making certain decisions will be helpful for making these arguments.

- Because each decision you make will involve different assumptions, document what you do and how it changes the conclusions you can make.

- One can also use multiple methods to address missing data. For example, one can start with listwise deletion to remove cases that are considered as having too much missing data or have missing data on variables that one does not want to impute. Then, Ml can be applied to the remaining cases.

- In case you're still worried, Graham and Schafer (1999) showed that MI performs very well in small samples (as low as $N=50$ ), even with very large multiple regression models (up to 18 predictors) and even with as much as $50 \%$ missing in the dependent variable (Graham, 2009). Beyond this, unless you have a very good reason not to adjust for missingness (i.e., it is probably not sufficient to just say "my data are MNAR" and not impute), you probably still want to use multiple imputation, FIML, or another missing data method. This is because not adjusting for missing values is a) wasteful under listwise deletion, and b) almost guarantees bias unless data are MCAR.

\section{- What software should I use?}

- Most statistical software has multiple imputation capabilities. However, very few imputation diagnostics have been made available in statistical software.

- R packages: mi, mice, and Amelia packages include features for model checking with core functions for imputing missing values (van Buuren \& Groothuis-Oudshoorn, 2011; Honaker et al., 2011; 
Su et al., 2011). VIM and miP packages visualise imputed data (Brix, 2012; Templ et al., 2015). These packages function to graphically compare distribution of observed and imputed data, by offering scatterplots for plotting observed and imputed data against another variable. The mi package has tools to produce residual plots to check imputation models when imputing data using MICE. Amelia software has a diagnostic feature called overimputation to produce cross-validation plots of mean imputed values against observed value with $90 \%$ confidence intervals (Honaker et al., 2011).

- There are very few imputation diagnostics available in SAS, Stata, SPSS, and Mplus which do not have built-in features for performing imputation diagnostics. (However, Stata and SAS do include checks of convergence, including trace plots and autocorrelation plots.)

- Although diagnostic features have not been incorporated into many statistical packages, it is possible to write syntax for these modelling checks.

- For example, in Stata, you can also use the user defined command midiagplots to produce graphical diagnostics. You can also compare imputed and observed data using plots such as kernel density plots.

- Example multiple imputation syntax for Stata, Mplus, SAS, SPSS, R, and Blimp is available at https://osf.io/j3f8m

- Here are some specific software resources:

- STATA

- https://stats.idre.ucla.edu/stata/seminars/mi_in_stata_pt1_ne w/ provides a helpful walk-through tutorial of multiple imputation

- "mibeta" is a user defined program that generates standardized coefficients for multiply imputed data 
- "micorr" is a program you can add in Stata to generate a correlation matrix for multiply imputed data sets:

https://www.stata.com/statalist/archive/2013-10/msg00730.ht $\underline{\mathrm{ml}}$

- "misum" to easily calculate summary statistics on imputed data.

- "mimrgns" is comparable to the native "margins" command and is compatible with margins plot:

https://www.stata.com/meeting/germany $16 /$ slides/de16 klein pdf

- "midiagplots" is a program to compare imputed data to observed data, see:

https://journals.sagepub.com/doi/pdf/10.1177/1536867X1201 $\underline{200301}$

- $\mathbf{R}$

- Several imputation packages are available in $\mathrm{R}$ (mice, mi, Amelia, imputeTS, hmi)

- Note that mice can now do multilevel imputation which likely makes it a better choice than $\mathrm{hmi}$. Both are limited to 2 level imputation models.

- A downside of $\mathrm{R}$ is that working with imputed data can be a bit trickier than actually imputing the data

- For example, you will need to use with() function to run regression models, and for() loops are useful for prepping all of the multiple imputation datasets.

- SAS

- Multiple Imputation of Complex Sample Data: https://support.sas.com/resources/papers/proceedings10/26 5-2010.pdf

- Sensitivity Analysis for MNAR: https://support.sas.com/resources/papers/proceedings14/SA S270-2014.pdf 
- Multiple Imputation and FIML for SEM:

https://support.sas.com/rnd/app/stat/papers/imps2011 FIML. pdf

\section{- Should I use multiple imputation or FIML?}

- Why is FIML an attractive alternative to multiple imputation?

- If you apply multiple imputation and FIML to the same input data under equivalent distributional assumptions, results will be essentially identical (Collins et al., 2001).

- According to Allison (2012):

- FIML is more efficient than multiple imputation. To be fully efficient, multiple imputation must produce and analyze an almost infinite number of datasets, whereas this is not the case with FIML (but, multiple imputation can get very close with a larger $m$ ).

- For a given set of data, FIML always produces the same result. However, multiple imputation can give a different result every time you use it (because it involves random draws and therefore produces different parameter estimates, standard errors, and test statistics). This raises the possibility that different investigators could reach different conclusions even when applying the same methods to the same data. (Note: This is a good justification for setting and reporting a seed number in multiple imputation to increase reproducibility!)

- FIML requires far fewer decisions than multiple imputation (e.g., imputation method overall and per variable, how many datasets to impute, how many iterations between datasets, prior distributions, incorporation of interactions and non-linearities, etc.).

- To include auxiliary variables in FIML with Mplus, you model them in your MODEL statement as autocorrelations, for example:

- Y1 Y2 Y3 with Y1 Y2 Y3; 
- This allows these variables to be used to help the FIML procedure even though they're not modeled elsewhere in your analyses.

- However, it can get finicky when you have many auxiliary variables to include.

- With multiple imputation, there may be conflict between the imputation model and the analysis model (e.g., if the analysis model contains variables that were not in the imputation model, or the imputation model is strictly linear but the analysis model contains interactions and non-linearities).

- This paper (https://arxiv.org/abs/1307.5875) argues that ML estimates have less bias than multiple imputation estimates, and that ML estimates have smaller sampling variance than multiple imputation estimates.

- However, there are several reasons why FIML may not be a good fit

- FIML will by default drop any cases where data are missing on your outcome variables. In some programs you can hack it to make it estimate, but not all.

- To our knowledge, FIML cannot accommodate multilevel models.

- Only parameters for the sample are estimated by FIML (variances, covariances), no values on a person or item level

- If you do not include auxiliary variables in FIML, it will underperform relative to multiple imputation when the data are not MCAR ( $\underline{\text { Collins }}$ et al., 2001).

- FIML does not allow for sensitivity analyses for MNAR data the way that multiple imputation does (e.g., see this example for SAS: https://statisticalhorizons.com/sensitivity-analysis)

- According to Enders (2010):

- Multiple imputation is the best at handling:

- Auxiliary variables

- Incomplete predictor variables

- Item-level missing data (i.e., psychometric scales) 
- FIML is the best at handling:

- Missing data in SEM

- Interaction effects (but this is only if you do not specify interaction effects in your MI model)

- Ease of use 
Table 3. Pros and Cons of FIML vs. Multiple Imputation

\begin{tabular}{|c|c|c|}
\hline & FIML & Multiple Imputation \\
\hline $\begin{array}{l}\text { What is your planned analytic } \\
\text { model? }\end{array}$ & $\begin{array}{c}\text { Best for SEM (and regression } \\
\text { by extension) }\end{array}$ & $\begin{array}{c}\text { Can handle any model (except } \\
\text { mixture models) }\end{array}$ \\
\hline $\begin{array}{l}\text { What if you have non-normal } \\
\text { data? }\end{array}$ & Specify robust standard errors & $\begin{array}{c}\text { Can address non-normal } \\
\text { variables - best to use MICE } \\
\text { (chained) }\end{array}$ \\
\hline $\begin{array}{l}\text { How many auxiliary variables } \\
\text { do you have? }\end{array}$ & \begin{tabular}{|c|} 
Easy for one or two; \\
Challenging for more (can more \\
easily include covariates \\
related to missingness in model \\
than auxiliary variables)
\end{tabular} & $\begin{array}{c}\text { Not really a concern because } \\
\text { algorithm operates no } \\
\text { differently from non-auxiliary } \\
\text { variables }\end{array}$ \\
\hline Can it handle multilevel data? & No & Yes \\
\hline How do you build models? & $\begin{array}{c}\text { Models are not nested (different } \\
\text { n's) so cannot use model fit } \\
\text { comparisons to check how well } \\
\text { it worked }\end{array}$ & $\begin{array}{c}\text { You can utilize model fit } \\
\text { statistics to determine how well } \\
\text { the imputation model fits your } \\
\text { data }\end{array}$ \\
\hline $\begin{array}{l}\text { How do you check } \\
\text { convergence issues? }\end{array}$ & $\begin{array}{c}\text { Need to check for negative } \\
\text { variances, etc. (SEM) }\end{array}$ & $\begin{array}{l}\text { Can be hard to gauge; need to } \\
\text { check trace plots, examine } \\
\text { each part of the model } \\
\text { separately to see where it fails }\end{array}$ \\
\hline Software Options & \begin{tabular}{|c|} 
Mplus, R lavaan (with \\
extensions), SAS, AMOS, Stata
\end{tabular} & $\begin{array}{c}\text { SPSS, SAS, Stata, Blimp, R } \\
\text { (mice, Amelia, mi, hmi) (with } \\
\text { extensions), Mplus }\end{array}$ \\
\hline
\end{tabular}




\section{References}

Allison, P. D. (2000). Multiple Imputation for Missing Data: A Cautionary Tale.

Sociological Methods \& Research, 28(3), 301-309.

https://doi.org/10.1177/0049124100028003003

Allison, P. D. (2002). Missing data. SAGE Publications, Inc. https://www.doi.org/10.4135/9781412985079

Allison, P. D. (2012). Handling Missing Data by Maximum Likelihood (working paper 312-2012). SAS Global Forum. https://statisticalhorizons.com/wp-content luploads/MissingDataByML.pdf

Azur, M. J., Stuart, E. A., Frangakis, C., \& Leaf, P. J. (2011). Multiple imputation by chained equations: What is it and how does it work? International Journal of Methods in Psychiatric Research, 20, 40-49. https://doi.org/10.1002/mpr.329

Baraldi, A. N., \& Enders, C. K. (2010). An introduction to modern missing data analyses. Journal of School Psychology, 48, 5-37. https://doi.org/10.1016/j.jsp.2009.10.001

Brix, P. (2012). miP: Multiple Imputation Plots. R package version 1.1. http://CRAN.R-project.org/package $=$ miP

Breiman, L., J. Friedman, R. Olshen, and C. Stone. 1984. Classification and Regression Trees. New York: Wadsworth Publishing.

Cham, H., Reshetnyak, E., Rosenfeld, B., \& Breitbart, W. (2017). Full information maximum likelihood estimation for latent variable interactions with incomplete indicators. Multivariate Behavioral Research, 52, 12-30. https://doi.org/10.1080/00273171.2016.1245600

Collins, L. M., Schafer, J. L., \& Kam, C. M. (2001). A comparison of inclusive and restrictive strategies in modern missing data procedures. Psychological Methods, 6(4), 330. https://www.researchgate.net/profile/Chi-Ming-Kam/publication/ 11578765 A Comparison of Restrictive Strategies in Modern Missing Data Procedures/links/02bfe510751ae2ec69000000/A-Comparison-of-Restrictive-Strat egies-in-Modern-Missing-Data-Procedures.pdf

Corker, K. S. (2021). An Open Science Workflow for More Credible, Rigorous Research (preprint). DOI: 10.31234/osf.io/wu6sn 
Croy, C. D., \& Novins, D. K. (2005). Methods for addressing missing data in psychiatric and developmental research. Journal of the American Academy of Child \& Adolescent Psychiatry, 44(12), 1230-1240. https://doi.org/10.1097/01.chi.0000181044.06337.6f

Enders, C. K. (2010). Applied missing data analysis. Guilford press. http://library.mpib-berlin.mpg.de/toc/z2010 1182.pdf

Enders, C. K., Baraldi, A. N., \& Cham, H. (2014). Estimating interaction effects with incomplete predictor variables. Psychological Methods, 19, 39-55. https://doi.org/10.1037/a0035314

Fielding, S., Fayers, P.M., McDonald, A., McPherson, G., \& Campbell, M.K. (RECORD Study Group) (2008). Simple imputation methods were inadequate for missing not at random (MNAR) quality of life data. Health Qual Life Outcomes, 6, 57. https://doi.org/10.1186/1477-7525-6-57

Freese, J. (2007). Replication standards for quantitative social science: Why not sociology? Sociological Methods \& Research, 36(2), 153-172. https://doi.org/10.1177/0049124107306659

Gelman, A., Carlin, J.B., Stern, H.S., Dunson, D.B., Vehtari, A., Rubin, D.B. (2013). Bayesian data analysis (3rd ed). Boca Raton: CRC Press. http://www.stat.columbia.edu/ gelman/book/

Gelman, A., King, G., \& Liu, C. (1998). Not asked and not answered: Multiple imputation for multiple surveys. Journal of the American Statistical Association, 93(443), 846-857. https://doi.org/10.1080/01621459.1998.10473737

Gelman, A., Van Mechelen, I., Verbeke, G., Heitjan, D. F., \& Meulders, M. (2005). Multiple imputation for model checking: completed-data plots with missing and latent data. Biometrics, 61, 74-85. https://doi.org/10.1111/j.0006-341X.2005.031010.x

Graham, J. W. (2009). Missing data analysis: Making it work in the real world. Annual Review of Psychology, 60, 549-576. https://doi.org/10.1146/annurev. psych.58.110405.085530

Graham, J. W., Olchowski, A. E., \& Gilreath, T. D. (2007). How many imputations are really needed? Some practical clarifications of multiple imputation theory. 
Prevention Science, 8(3), 206-213.

https://psycnet.apa.org/doi/10.1007/s11121-007-0070-9

Graham, J. W., \& Schafer, J. L. (1999). On the performance of multiple imputation for multivariate data with small sample size. In R. Hoyle (Ed.), Statistical strategies for small sample research (pp. 1-29). Thousand Oaks, CA: Sage.

He, Y., \& Zaslavsky, A. M. (2012). Diagnosing imputation models by applying target analyses to posterior replicates of completed data. Statistics in Medicine, 31, 1-18. https://doi.org/10.1002/sim.4413

Heeringa, S., West, B. T., \& Berglund, P. A. (2010). Applied survey data analysis. Boca Raton, FL: Chapman \& Hall. https://doi.org/10.1201/9781315153278

Hernán, M. A., Hernández-Díaz, S., \& Robins, J. M. (2004). A structural approach to selection bias. Epidemiology, 615-625. https://www.jstor.org/stable/20485961

Honaker, J., King, G., \& Blackwell, M. (2011). Amelia II: A program for missing data. Journal of statistical software, 45, 1-47. https://www.jstatsoft.org/article/view/v045i07

Johnson, D. R., \& Young, R. (2011). Toward best practices in analyzing datasets with missing data: Comparisons and recommendations. Journal of Marriage and Family, 73(5), 926-945. https://doi.org/10.1111/j.1741-3737.2011.00861.x

Kenward, M. G., \& Carpenter, J. (2007). Multiple imputation: current perspectives. Statistical Methods in Medical Research, 16(3), 199-218. https://doi.org/10.1177/0962280206075304

King, K. M., Kim, D. S., \& McCabe, C. J. (2018). Random responses inflate statistical estimates in heavily skewed addictions data. Drug and Alcohol Dependence, 183, 102-110. https://doi.org/10.1016/j.drugalcdep.2017.10.033

Lee, S. Y., \& Song, X. Y. (2004). Evaluation of the Bayesian and maximum likelihood approaches in analyzing structural equation models with small sample sizes. Multivariate Behavioral Research, 39(4), 653-686. https://doi.org/10.1207/s15327906mbr3904 4

Light, R. J., Singer, J. D., \& Willett, J. B. (1990). By design: Planning research on higher education. Harvard University Press. 
Liu, Y., \& Sriutaisuk, S. (2021). A comparison of FIML- versus multiple-imputation-based methods to test measurement invariance with incomplete ordinal variables.

Structural Equation Modeling: A Multidisciplinary Journal, 1-19.

https://doi.org/10.1080/10705511.2021.1876520

Mack, C., Su, Z., Westreich, D. (2018). Types of missing data. Managing Missing Data in Patient Registries: Addendum to Registries for Evaluating Patient Outcomes: A User's Guide (3rd ed). Rockville: Agency for Healthcare Research and Quality (US). https://www.ncbi.nlm.nih.gov/books/NBK493614/

Madley-Dowd, P., Hughes, R., Tilling, K., \& Heron, J. (2019). The proportion of missing data should not be used to guide decisions on multiple imputation. Journal of Clinical Epidemiology, 110, 63-73. https://doi.org/10.1016/j.jclinepi.2019.02.016

Manly, C. A. \& Wells, R. S. (2015). Reporting the use of multiple imputation for missing data in higher education research. Research in Higher Education, 56, 397-409. https://doi.org/10.1007/s11162-014-9344-9

McKnight, P. E., McKnight, K. M., Sidani, S., \& Figueredo, A. J. (2007). Missing data: A gentle introduction. Guilford Press.

Mertens, G., \& Krypotos, A. M. (2019). Preregistration of analyses of preexisting data. Psychologica Belgica, 59(1), 338. https://dx.doi.org/10.5334\%2Fpb.493

Multiple Imputation in Stata. UCLA: Statistical Consulting Group. https://stats.idre.ucla.edu/stata/seminars/mi_in_stata_pt1_new/

Newman, D. A. (2014). Missing data: Five practical guidelines. Organizational Research Methods, 17(4), 372-411. https://doi.org/10.1177/1094428114548590

Nguyen, C. D., Lee, K. J., \& Carlin, J. B. (2015). Posterior predictive checking of multiple imputation models. Biometrical Journal, 57(4), 676-694. https://doi.org/10.1002/bimj.201400034

Nosek, B. A., Ebersole, C. R., DeHaven, A. C., \& Mellor, D. T. (2018). The preregistration revolution. Proceedings of the National Academy of Sciences, 115(11), 2600-2606. https://doi.org/10.1073/pnas.1708274114

Open Science Collaboration. (2015). Estimating the reproducibility of psychological science. Science, 349(6251). https://doi.org/10.1126/science.aac4716 
Peugh, J. L., \& Enders, C. K. (2004). Missing data in educational research: A review of reporting practices and suggestions for improvement. Review of Educational Research, 74(4), 525-556. https://doi.org/10.3102/00346543074004525

Royston, P., \& White, I. R. (2011). Multiple imputation by chained equations (MICE): implementation in Stata. J Stat Softw, 45(4), 1-20. https://onlinelibrary.wiley.com/doi/pdf/10.1002/sim.4067

Rubin, D. B. (1987). Multiple imputation for nonresponse in surveys. New York: Wiley. https://onlinelibrary.wiley.com/doi/pdf/10.1002/9780470316696.fmatter

Schafer, J. L. (1997). Analysis of incomplete multivariate data. London: Chapman \& Hall.

Schafer, J. L. (1999). Multiple imputation: a primer. Statistical methods in medical research, 8(1), 3-15. https://doi.org/10.1177/096228029900800102

Schafer, J. L., \& Graham, J. W. (2002). Missing data: our view of the state of the art. Psychological Methods, 7(2), 147. https://doi.org/10.1037/1082-989X.7.2.147

Schafer, J. L., \& Olsen, M. K. (1998). Multiple imputation for multivariate missing-data problems: A data analyst's perspective. Multivariate behavioral research, 33(4), 545-571. https://doi.org/10.1207/s15327906mbr3304_5

Social Science Computing Cooperative (2012). Multiple imputation in Stata: Introduction. University of Wisconsin, Madison. http://www.ssc.wisc.edu/sscc/pubs/stata mi intro.htm.

Stuart, E. A., Azur, M., Frangakis, C., \& Leaf, P. (2009). Multiple imputation with large data sets: a case study of the Children's Mental Health Initiative. American Journal of Epidemiology, 169(9), 1133-1139. https://doi.org/10.1093/aje/kwp026

Su, Y. S., Gelman, A., Hill, J., \& Yajima, M. (2011). Multiple imputation with diagnostics (mi) in R: Opening windows into the black box. Journal of Statistical Software, 45, 1-31. https://www.jstatsoft.org/article/view/v045i02

Sullivan, G. M., \& Feinn, R. (2012). Using effect size-or why the P value is not enough. Journal of Graduate Medical Education, 4(3), 279-282. https://doi.org/10.4300/JGME-D-12-00156.1

Tabachnick, B.G. \& Fidell, L.S. (2012) Using Multivariate Statistics (6th Ed.), Boston: Pearson Education. 
Taylor, L., \& Zhou, X. H. (2009). Relaxing latent ignorability in the ITT analysis of randomized studies with missing data and noncompliance. Statistica Sinica, 749-764. https://www.jstor.org/stable/24308854

Templ, M., Alfons, A., Kowarik, A., Prantner, B. (2015). VIM: Visualization and Imputation of Missing Values. R package version 4.3.0, http://CRAN.R-project.org/package=VIM

Tierney, W., Hardy III, J. H., Ebersole, C. R., Leavitt, K., Viganola, D., Clemente, E. G., ... \& Hiring Decisions Forecasting Collaboration. (2020). Creative destruction in science. Organizational Behavior and Human Decision Processes, 161, 291-309. https://doi.org/10.1016/j.obhdp.2020.07.002

Tierney, W., Hardy III, J., Ebersole, C. R., Viganola, D., Clemente, E. G., Gordon, M., ... \& Culture \& Work Morality Forecasting Collaboration. (2021). A creative destruction approach to replication: Implicit work and sex morality across cultures. Journal of Experimental Social Psychology, 93, 104060. https://doi.org/10.1016/j.jesp.2020.104060

Treiman, D. J. (2009). Quantitative data analysis: Doing social research to test ideas. San Francisco: Jossey-Bass.

Tsiampalis, T., \& Panagiotakos, D.B. (2020). Missing-data analysis: Socio-demographic, clinical and lifestyle determinants of low response rate on self- reported psychological and nutrition related multi-item instruments in the context of the ATTICA epidemiological study. BMC Med Res Methodol, 20, 148. https://doi.org/10.1186/s12874-020-01038-3

Van Buuren, S. (2007). Multiple imputation of discrete and continuous data by fully conditional specification. Statistical Methods in Medical Research, 16(3), 219-242. https://doi.org/10.1177/0962280206074463

van Buuren, S. (2018). Flexible Imputation of Missing Data (2nd edition). Chapman \& Hall CRC Press. https://stefvanbuuren.name/fimd/

Van Buuren, S., Brand, J. P., Groothuis-Oudshoorn, C. G., \& Rubin, D. B. (2006). Fully conditional specification in multivariate imputation. Journal of Statistical Computation and Simulation, 76(12), 1049-1064. https://doi.org/10.1080/10629360600810434 
van Buuren, S., \& Groothuis-Oudshoorn, K. (2011). mice: Multivariate imputation by chained equations in R. Journal of Statistical Software, 45, 1-67. http://dx.doi.org/0.18637/jss.v045.i03

van Ginkel, J.R., Linting, M., Rippe, R.C.A., \& van der Voort, A. (2020). Rebutting existing misconceptions about multiple imputation as a method for handling missing data. Journal of Personality Assessment, 102(3), 297-308. https://doi.org/10.1080/00223891.2018.1530680

Von Hippel, P. (2016). The number of imputations should increase quadratically with the fraction of missing information (arXiv preprint). http://hbiostat.org/papers/missingData/hip16num.pdf

von Hippel, P. T. (2018). HOW_MANY_IMPUTATIONS: Stata module to determine required number of imputations (working paper). https://EconPapers.repec.org/RePEc:boc:bocode:s458452

White, I. R., Royston, P., \& Wood, A. M. (2011). Multiple imputation using chained equations: issues and guidance for practice. Statistics in Medicine, 30(4), 377-399. https://doi.org/10.1002/sim.4067

Young, R., \& Johnson, D. R. (2010). Imputing the missing Y's: implications for survey producers and survey users. In Proceedings of the AAPOR conference abstracts (pp. 6242-6248). https://www.researchgate.net/profile/David-Johnson-108 Ipublication/266357732 Imputing the Missing Y\%27s Implications for Survey Producers_and_Survey_Users/links/54c1237f0cf2dd3cb95804bc/lmputing-theMissing-Ys-Implications-for-Survey-Producers-and-Survey-Users.pdf 